\title{
A CFD/DEM Approach to Determine the Flow Resistance of Randomly Packed Bed of Crushed Rock Particles
}

\author{
Jaap Hoffmann \\ Dept. of Mechanical \& Mechatronic Engineering, Stellenbosch University \\ Private Bag X1, Matieland, 7602, South Africa \\ hoffmaj@sun.ac.za
}

\begin{abstract}
Packed beds of crushed rock particles are typically encountered in the thermal energy storage system at a solar thermal power plant. Rocks have irregular shapes, and tend to pack with their long axes in a horizontal plane. As a results its resistance to airflow is anisotropic. Designing a bed for effective charging and discharging cycles, depends on an accurate prediction of the flow distribution through it. Ellipsoids are mathematically simple shapes that characterise crushed rock particles reasonably successfully. A packed bed was generated using a discrete element model, and the flow through the bed calculated with computational fluid dynamics. The resistance tensor for the bed was extracted from the data. The tensor is almost symmetrical, with the diagonal terms dominant. Incorporating this tensor as a source term in a porous medium presentation of a packed bed, should yield more accurate results than, say the Ergun equation, at very little extra computational overhead.
\end{abstract}

Keywords: Packed bed; anisotropic resistance; CFD/DEM coupling; thermal energy storage

\section{Introduction}

Most of the world's electricity is derived from burning fossil fuels. South Africa is an extreme example, with $80 \%$ of its electricity is generated from coal [1]. This is not a sustainable situation, as fossil fuels are a limited resource that is rapidly being depleted, which contributes to air pollution and greenhouse gas emissions. The South African government has set a target to reduce its reliance on coal to $30 \%$ of the energy mix by 2050 [1]. Renewable energy is expected to replace coal in the country's energy mix.

South Africa has one of the best solar resources in the world, a moderate wind resource but limited hydro and biomass resources [2]. Solar photovoltaic (PV) power and concentrated solar thermal power (CSP) are the two main competing technologies to fill the void in the energy mix. Solar PV is cheaper to generate, but large scale battery storage is not sufficiently developed to ensure continuous supply throughout the night. CSP with thermal energy storage is a proven dispatchable electricity generation technology, but comes at a higher cost.

Solarized Brayton cycles attracted attention due to their inherent high efficiency at high temperature, the absence of a phase change, the option to combine it with a bottoming Rankine cycle and the ease of storing waste heat in an inexpensive rock bed thermal energy storage [3]. To reduce the cost even further, it is necessary to design the rock bed storage in such a way that the thermal capacity of the bed is maximized, whilst pumping costs are minimized.

Packed beds of crushed rock particles are anisotropic [4 - 6], with the resistance tensor depending on particle size, shape, orientation and packing density. Small beds for thermal energy storage as encountered in building comfort applications, and laboratory-scale solar plant, usually comprise of a bed contained in a prismatic cylinder subject to plug flow [7]. Bed resistance is determined experimentally, with possible adjustments for a lower packing density at the containment walls. The latter is of importance in the design of chemical or nuclear reactors, where local reaction rates and/or energy release is affected by local packing (area) density and fluid flow rates through the bed [8].

Irregular particles are most often represented by equivalent spheres [9]. Mass/volume equivalent diameter is easier to measure, but when surface reactions are a key performance indicator, the surface equivalent diameter might be a more appropriate parameter. Other shape indicators, such as sphericity, aspect ratio and roundness, were introduced to improve the correlation between experimental data and models with some success. Although spheres are the most popular shape used in modelling packed beds, it fails to mimic the anisotropic nature of packed beds of irregular particles. More realistic results were reported from modelling particles as monodisperse cylinders, ellipsoids or rectangular prisms [10 - 12]. 
Extracting information on packing structure and flow through a rock bed relies on expensive and time-consuming techniques such as X-ray tomography and particle image velocimetry [13]. Most researchers adopted discrete element modelling (DEM) and computational fluid dynamics (CFD) simulations for this purpose [14, 15]. Early DEM models placed particles randomly in a container, and perturbed their positions and orientations until their geometries don't overlap. Physically more realistic models took the contact forces between particles into account.

Meshing contact points between particles in CFD models proved troublesome; shrinking or enlarging particles change the porosity of the bed, whilst building bridges affect only the contact zone between particles where the flow is almost stagnant [16]. Modelling the flow in the interstitial volumes is limited to a few hundred particles due to hardware constraints. Simulations focus mainly on (transition to) turbulence [17 - 19], using direct numerical simulation (DNS) or lattice-Boltzmann methods, although [17] reported reasonable results from Reynolds averaged Navier-Stokes (RANS) models. Steady-state RANS models make it practically achievable to model a large number of particles, in different random packing configurations, and at different flow velocities and directions.

This paper addresses the characterization of crushed dolerite rock particles, a DEM simulation of randomly packed beds of said particles, the CFD simulation of the flow through the voids, experimental validation of the models, and the extraction of the resistance tensor from simulation results.

\section{Problem statement}

Nuclear and chemical reactors typically comprise a prismatic container filled with a randomly packed bed of spherical or irregular particles. Despite the random packing of the particles, plug flow (with compensation for the near-wall region) can be assumed in most cases. Resistance coefficients can be determined experimentally. In contrast, the size of rock bed thermal energy storage facilities for utility-scale solar thermal power plants, means that the bed has a frustum shape, with multiple in- and outlets. Irregular particle size leads to anisotropic resistances in the horizontal and vertical directions. The flow is fully three dimensional, and is subject to bed anisotropy. To date, limited research has been done on 3D flow through anisotropic beds of crushed rock particles.

\section{Dimensional analysis}

According to $[5,20]$, the pressure drop per unit length (source term) across an infinite, anisotropic packed bed is:

$S_{i}=-\left\{\sum_{j=1}^{3} D_{i j} \mu v_{j}+\sum_{j=1}^{3} C_{i j} \frac{1}{2} \rho|\vec{V}| v_{j}\right\}$

$\ldots$ with $S_{i}$ a momentum source term per unit volume. The coefficients of the viscous $\left(D_{i j}\right)$ and inertial $\left(C_{i j}\right)$ tensors should depend on particle characteristic lengths, $L_{M}, L_{I}$ and $L_{S}$, packing density $\psi$ or porosity $\varepsilon(\varepsilon=1-\psi)$, packing coordination number (average number of contacts) $\beta$, surface roughness $\epsilon$, as well as fluid density $\rho$ and viscosity $\mu$, and a fluid velocity. If one assumes a priori particles of an ellipsoidal shape, $L_{M}, L_{I}$ and $L_{S}$ can be replaced by the semi-major and minor axes, $a, b$, and $c$. Laser scanning of a few particles suggest that the surface area of the mass equivalent ellipsoidal particle underestimates the surface area of the real particles by $10-15 \%$. A modified sphericity $\phi$ was introduced to accommodate this difference. The definition of the superficial velocity, $V_{s}$, is fairly unambiguous, and is adopted here. Dimensional analysis suggests that the resistance tensors should depend on

$$
C_{i j}=f\left\{\left(\frac{\rho V_{s} D_{p}}{\mu \varepsilon}\right),\left(\frac{\epsilon}{D_{p}}\right),\left(\frac{a}{c}\right), \varepsilon, \phi, \beta, \theta_{i}\right\}
$$

Li et al [21] suggest that Herman's orientation factor, $H_{i}$, should replace the particle orientation angle.

$$
H_{i}=\frac{\left\{3 \overline{\cos ^{2} \theta_{l}}-1\right\}}{2}
$$

A value of $H_{i} \approx 1$ means almost perfect alignment, whilst $H_{i} \approx 0$ indicates no alignment at all. The modified sphericity, $\phi$, compares the surface area of actual particles, $A_{p}$, to the surface area $A_{e}$ of their mass equivalent ellipsoidal representations. Calculating the exact surface area of an ellipsoid involves elliptical integrals. A simplified expression for the surface of an ellipsoidal particle, that is accurate to within $1 \%$, is given by Spiegel [22]

$$
A_{e} \approx 4 \pi\left\{\frac{(a b)^{p}+(a c)^{p}+(b c)^{p}}{3}\right\}^{1 / p}
$$


According to Spiegel [22], the best results are achieved for $p=1.607$.

\section{Sampling}

A truckload of crushed dolerite rock (railway ballast, passing through a $75 \mathrm{~mm}$ sieve [23]), was available for testing. More than 100 particles were collected randomly from the rock pile, using a shovel and bucket. The dimensions of the bounding box for each particle was measured using a Vernier calliper, and their mass was determined to within 0.01 gram, using an electronic scale. Bagheri et al [11] estimate that the average operator error using calliper measurements, as illustrated in figure 1(b), is about $5 \%$. It was assumed that the particles are large enough for the particle density to remain constant at $2900 \mathrm{~kg} / \mathrm{m}^{3}$ [24].

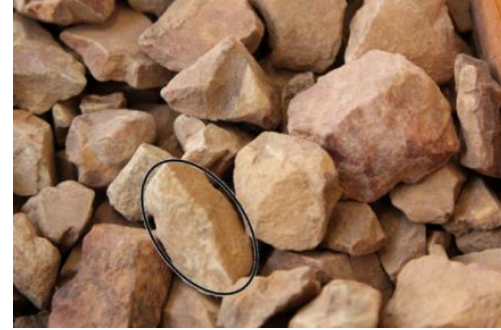

(a)

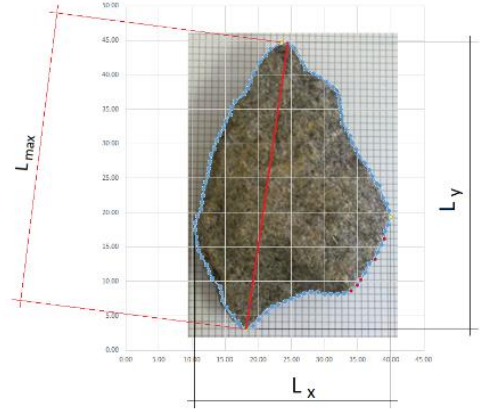

(b)

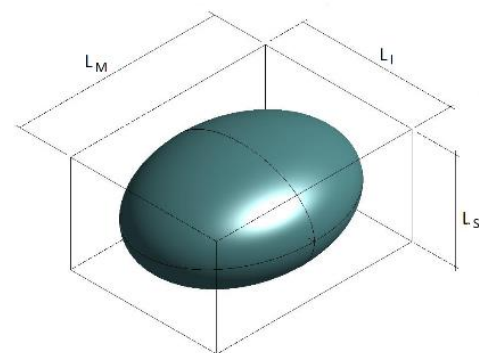

(c)

Figure 1. Sampling of particles, and representative particle (right).

Moving averages of particle dimensions are shown in Figure 2. The volume equivalent diameter converged within about 40 particles, whilst the long, intermediate and short axes reached constant values after about 75 particles were measured. The representative ellipsoidal particle, as shown in figure 1(c), has $a=87.9 \mathrm{~mm}, b=59.5 \mathrm{~mm}$ and $c=37.4 \mathrm{~mm}$ and the diameter of the volume equivalent sphere is $55.2 \mathrm{~mm}$, based upon a mean particle density of $2600 \mathrm{~kg} / \mathrm{m}^{3}$.

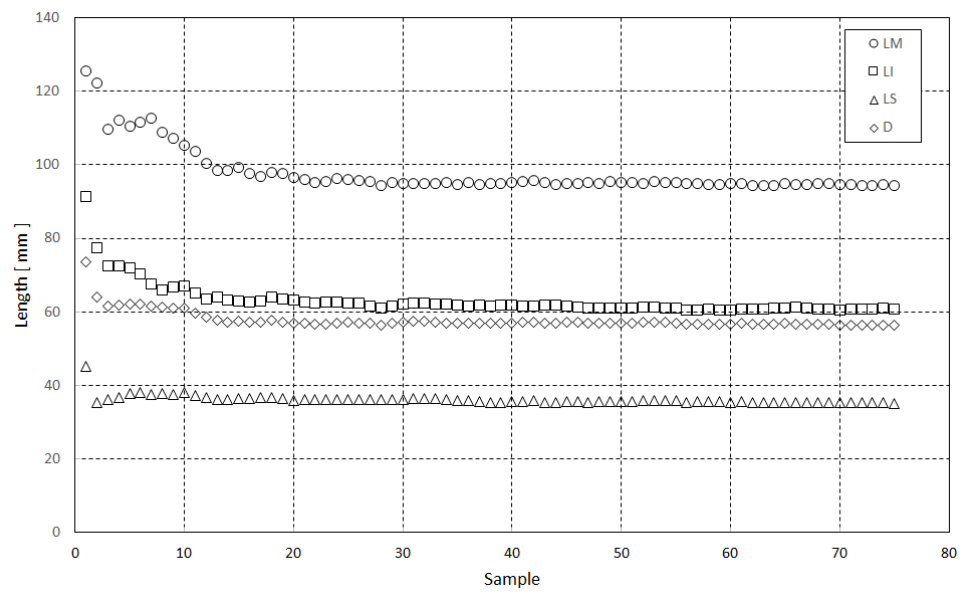

Figure 2. Moving averages of long, intermediate and short axes of particles, as well as volume equivalent diameter.

\section{Numerical work}

\subsection{Discrete element modelling (DEM)}

Monodispersed, faceted elliptical particles were dropped from $2 \mathrm{~m}$ height into a spherical container measuring 1.142 $\mathrm{m}$ in diameter, using the commercial DEM code Rocky 4.1, as shown in figure 3. Particles were dropped with their long axes aligned with the vertical (or y-direction), and their short axes aligned with the z-direction. The energy released from 
repeated impacts from particles dropping into the cage resulted in a random closed packing with a void fraction of $34 \%$. The cage was allowed to overflow, and particles accumulating over and above the inlet of the sphere was removed in the CFD code. Lommen et al [25] found that reducing Young's modulus of the particles by a few orders of magnitude, has an insignificant influence on the packing structure/density, but speed up the DEM simulations significantly. Furthermore, a slightly softer material allows inter-penetration of contacting particles, changes point contacts into plane contacts. This allows for easier meshing in the CFD code. It is offered here as an alternative to the usual methods of shrinking or enlarging particles, bridging contact points, or immersed boundary methods [26] to overcome meshing problems.

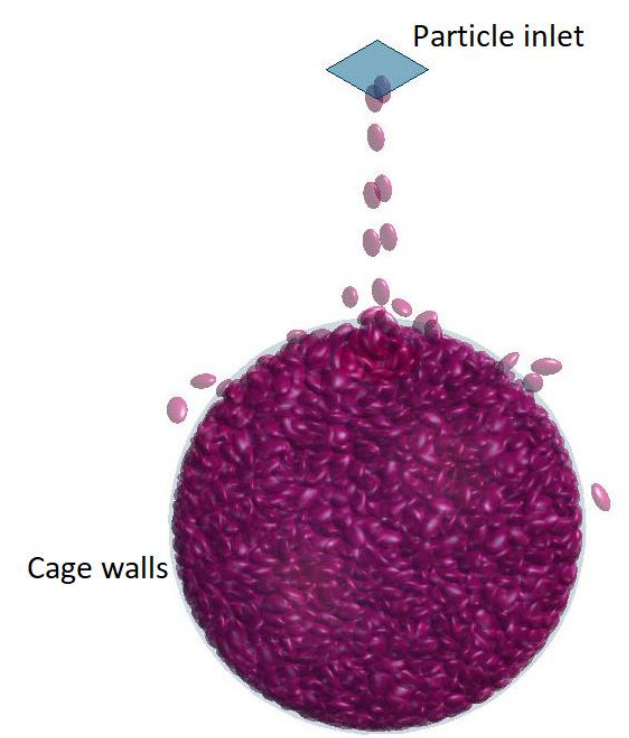

Figure 3. Particle positions for the filled cage.

The output from the DEM code includes amongst others the particle position vector, particle orientation (given as a rotation axis and angle of rotation around said axis) and the number of contacts. From this information, it is possible to obtain the particle coordination number and Herman's orientation factor [21]. The most useful expression of the latter was in terms of the short axis $-H_{S y}=0.519$. A plausible explanation for this is that the particles are relatively flat, and tend to settle with the plane formed by the long and intermediate axes aligned with the horizontal. It is evident from the near isotropic distribution of the angles between, say the $\mathrm{x}$-axis and the long axis of the ellipsoid $\left(H_{L x}=0.026\right)$, that the long axis does not show a preference for any direction in the horizontal plane. The coordination number (number of contacts) is not meaningful when one looks at monodispersed particles.

\subsection{Computational fluid dynamics (CFD)}

An ellipsoidal particle was created in ANSYS SpaceClaim. Particles were first rotated by the rotation angle about the rotation vector, and then copied to the positions obtained from the DEM code Rocky 4, to fill the same sized spherical cage that was used in the tests. The solid particles were subtracted from a rectangular slice along the centre of the wind tunnel, to obtain the interstitial flow domain only, as shown in figure 4 . The slice was rotated in steps of $15^{\circ}$ degrees about its azimuthal angle $\phi$ and elevation angle $\theta$, such that an entire octant is covered.

Each combination of $\phi$ and $\theta$ constitutes a CFD case, that was simulated over a pore Reynolds number range of $60-$ 2 000, for a total of 294 simulations. The Reynolds number range was chosen to overlap the experimental data of Hoffmann and Lindeque [27] (for validation purposes), but also to augment the experimental data at the lower end of the scale. For porous media, transition to turbulent flow occurs at about $\operatorname{Re}_{\text {pore }} \sim 350$ [18], with

$$
D_{\text {pore }}=\frac{2}{3}\left(\frac{\varepsilon}{1-\varepsilon}\right) D_{p}
$$




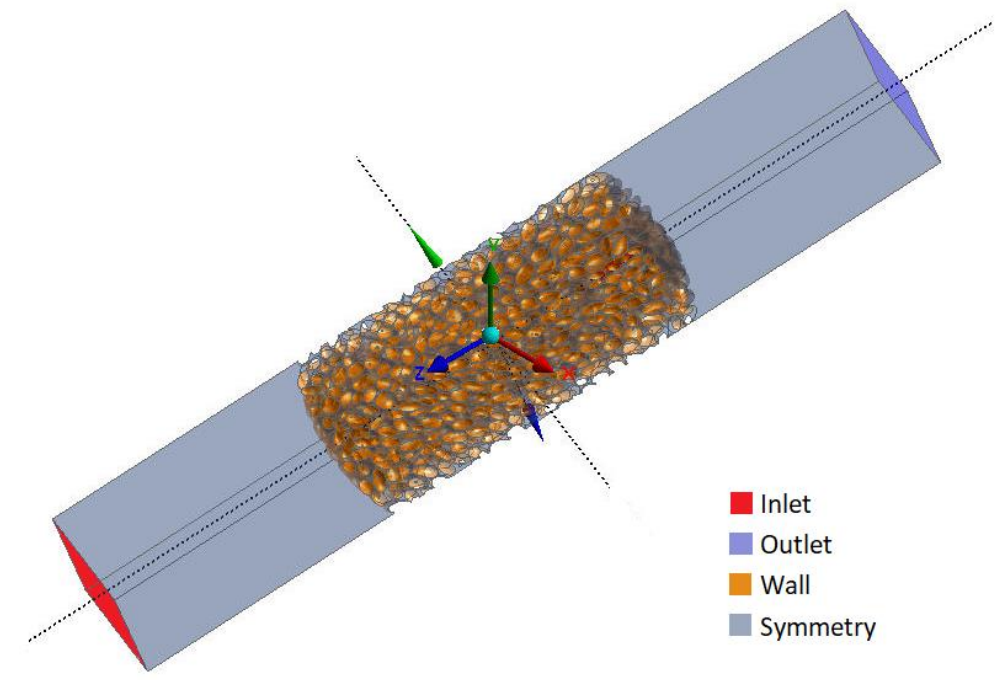

Figure 4. Computational domain

Hence, all but the three lowest Reynolds number case will be turbulent. Computational resources to our disposal did not allow the use of the more appropriate large eddy simulation or low Reynolds number RANS models. We followed the lead of Linsong et al [17] and adopted a high Reynolds number $k$ - $\varepsilon$ turbulence model with scalable wall functions to compensate for the large differences in, and lack of user control over, $y^{+}$values. It is expected that the frequent changes in flow direction and/or velocity due to the tortuous flow paths through the pores will dominate the pressure drop, and that the prediction of viscous drag is of secondary importance.

The sidewalls of the domain were modelled as symmetry planes. This is not geometrically correct in the case of a randomly packed bed, but hardware limitations ruled out modelling the entire domain. To quantify the effect of these boundary conditions, simulations were done for a domain that is about $4 \mathrm{D}_{\mathrm{p}}$ square, $6 \mathrm{D}_{\mathrm{p}}$ square and $8 \mathrm{D}_{\mathrm{p}}$ square. Results show that after $6 \mathrm{D}_{\mathrm{p}}$ across, the effect of the symmetry boundary on the overall pressure drop becomes insignificant.

\subsubsection{Model and Mesh}

A tetrahedral dominant mesh, with a maximum cell size of $5 \mathrm{~mm}$, was constructed in ANSYS meshing. Curvature and proximity size functions, with a minimum cell size of $0.5 \mathrm{~mm}$, and a $10^{\circ}$ angle between face normals on curved surfaces, were used. Typical cell count of a $6 \mathrm{D}_{\mathrm{p}}$ mesh is 60 million cells. Converted to polyhedra, the cell count reduces to about 15 million cells. Due to the acute angles between contacting particles, it was necessary to bridge contacts between some cells, inserting a $5 \mathrm{~mm}$ diameter sphere at the centre point of the contact surface.

A constant velocity normal to the cell faces is prescribed at the inlet boundary, whilst the outlet is a constant pressure outlet boundary. Symmetry boundary conditions are prescribed at the sides of the domain, and stationary wall conditions ate the particle/fluid interface. Due to the low flow velocities, it is assumed that the fluid (air) is incompressible with constant density and dynamic viscosity of $1.2 \mathrm{~kg} / \mathrm{m}^{3}$ and $1.89 \times 10^{-5} \mathrm{~Pa}$.s respectively.

Relaxation factors of 0.2 and 0.5 respectively for pressure and momentum equations were required to ensure convergence. Flow variables for highly skewed cells were calculated from the average of their neighbouring cells, rather than fluxes across the cells faces. These cells are invariably in the dead zones near particle/particle contacts.

The pressure drop across the domain was monitored, and the solution terminated if the pressure drop does not vary by more than $0.2 \%$ from its average for the last 1000 iterations. Residuals do not converge by more than one order of magnitude for pressure, and two orders of magnitude for velocity, but they stabilize at their low values after about 500 iterations. A "converged" solution is declared after 2000 iterations if all the above criteria are met. Run time is less than 1 hour on a 72 processor Xeon cluster. 


\subsubsection{Validation and Verification}

Verification comprises comparing results on coarse, medium and fine meshes. All meshing parameters are decreased by $20 \%$ between the coarse and medium, and again between the medium and fine meshes. Full mesh independence could not be claimed, due to hardware restrictions (a $64 \mathrm{~GB}, 10$ processor machine for meshing purposes), but the percentage error in pressure drop between the fine and coarse mesh, $\left(\Delta P_{f}-\Delta P_{c}\right) / \Delta P_{f}=0.17 \%$.

Validation is claimed through earlier work by Hoffmann and Lindeque [27], doing DEM/CFD and experiments on the same particles packed randomly in a square container. Their test and simulation data agree to within $9 \%$.

\section{Results and Discussion}

A plot of the pressure drop for an inlet velocity $U_{i}=0.2 \mathrm{~m} / \mathrm{s}$ is shown in figure 5 . It is clear that the altitude angle $\theta$ is the main contributor to pressure drop. This is consistent with the hypothesis that the particles tend to align with their long axes in a horizontal plane, as indicated by the high value of Herman's orientation factor. Figure 5 hints at symmetry about an azimuthal angle of $45^{\circ}$, but the change in pressure drop with azimuthal angle $\phi$ is much smaller than the change with altitude angle.

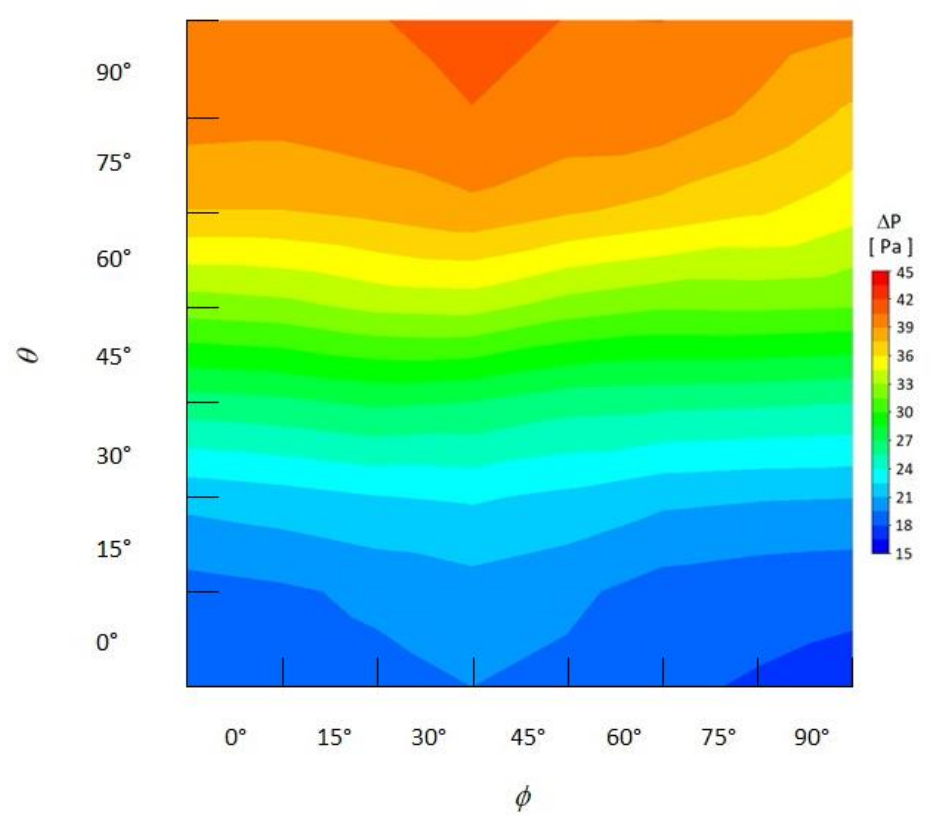

Figure 5. Pressure drop across the bed as a function of azimuthal and elevation angles.

The coefficients of the resistance tensor were obtained from data regression on the simulation results. Correlation coefficients $\left(R^{2}\right)$ were $0.96,0.98$ and 0.96 for equations $(6),(7)$ and (8) respectively.

$$
\begin{aligned}
& S_{x}=\frac{\Delta P}{L} \cos \theta \cos \phi=\mu u_{x} D_{x x}+\mu u_{y} D_{x y}+\mu u_{z} D_{x z}+\frac{1}{2} \rho|u|\left\{u_{x} C_{x x}+u_{y} C_{x y}+u_{z} C_{x z}\right\} \\
& S_{y}=\frac{\Delta P}{L} \sin \theta=\mu u_{x} D_{y x}+\mu u_{y} D_{y y}+\mu u_{z} D_{y z}+\frac{1}{2} \rho|u|\left\{u_{x} C_{x y}+u_{y} C_{y y}+u_{z} C_{y z}\right\} \\
& S_{z}=\frac{\Delta P}{L} \cos \theta \cos \phi=\mu u_{x} D_{z x}+\mu u_{y} D_{z y}+\mu u_{z} D_{z z}+\frac{1}{2} \rho|u|\left\{u_{x} C_{z x}+u_{y} C_{z y}+u_{z} C_{z z}\right\}
\end{aligned}
$$

$L$ is the length of the bed $\left(L \approx D_{c}\right)$, and $\mu$ and $\rho$ the dynamic viscosity and density of air respectively. The viscous and inertial resistance tensors are given by equations (9) and (10) below. It is clear that the diagonal terms are dominant, and that the tensors are approximately symmetrical $\left(D_{i j} \approx \pm D_{j i}\right.$ for non-diagonal terms). The high flow resistance in the $y$ direction is reflected in $D_{y y}$ and $C_{y y}$ being about $60 \%$ higher than the other diagonal terms. 


$$
D_{i j}=\left[\begin{array}{ccc}
2850271 & 301815 & -97715 \\
-342483 & 4468018 & -331956 \\
-119096 & 295676 & 2897912
\end{array}\right]
$$

... and

$$
C_{i j}=\left[\begin{array}{ccc}
500.235 & 72.636 & -34.007 \\
-83.235 & 905.835 & -111.225 \\
-16.297 & 70.199 & 470.009
\end{array}\right]
$$

The numerical values of the coefficients are about $40 \%$ higher than those reported by Hoffmann and Lindeque [27]. This difference can be explained by the volume of the cage in the current study being almost three times larger than that used by Hoffmann and Lindeque. In the smaller cage, walls effects and an overall lower packing density is expected to lower the flow resistance.

\section{Conclusion}

Viscous and inertial resistance coefficients for a randomly packed bed of crushed rock were extracted from a discrete element and computational fluid dynamic modelling. An ellipsoid, with the same volume and aspect ratio as the average crushed rock particle, was chosen as the representative particle. Using a larger cage, reduced wall effects and increased packing density. The resistance tensor is fairly symmetrical, with diagonal terms an order of magnitude larger than nondiagonal terms. Diagonal coefficients in the y-direction (vertical, relative to original pour direction) are about $60 \%$ larger than non-diagonal coefficients. Using the anisotropic resistance tensor should enable researchers to predict local hot spots and dead zones in a rock bed thermal energy storage facility fairly accurately. Experimental validation is planned for the first semester of 2020, as well as further DEM/CFD simulations to introduce statistical variation into the results.

\section{Acknowledgement}

The authors acknowledge the Centre for High-Performance Computing (CHPC), South Africa, for providing computational resources to this research project.

\section{References}

[1] Anonymous, Integrated Resource Plan (IRP2019), "South African Department of Energy", October 2019, http://www.energy.gov.za/files/docs/IRP\%202019.pdf, accessed (28/11/2019).

[2] J. Calitz and J. Wright, "Statistics of utility-scale solar PV, wind and CSP in South Africa in 2018", https://researchspace.csir.co.za/ dspace/bitstream/handle/10204/10636/Calitz_21959_2019.pdf?sequence=1\&isAllowed=y, accessed 28/11/2019.

[3] K. Ellingwood, S.M. Safdarnejad, K. Rashid and K. Powell, "Leveraging energy storage in a solar-tower and combined cycle hybrid power plant", Energies, vol. 12, No. 1, pp. 1 - 23, 2019.

[4] K.G. Allen, T.W. van Backström and D.G. Kröger, "Packed bed pressure drop dependence on particle shape, size distribution, packing arrangement and roughness", Powder Technology, vol. 246, pp. 590 - 600, 2013.

[5] S.A. Galindo-Torres, A. Scheuermann and L. Li, "A numerical study on the permeability in a tensorial form for laminar flow in anisotropic porous media", Physical Review E, vol. 86, pp. 046306-1 to 046306-9, 2012.

[6] P. Guo, "Dependency of tortuosity and permeability of porous media on directional distribution of pore voids", Transport in Porous Media, vol. 95, pp. 285 - 303, 2012.

[7] A. Jafari, P. Zamankhan, S.M. Mousavi and K. Pietarinin, "Modeling and CFD simulation of flow behaviour and dispersivity through randomly packed bed reactors", Chemical Engineering Journal, vol. 144, pp. 476 - 482, 2008.

[8] M. de Beer, P.G. Rousseau and C.G. du Toit, "A review of methods to predict the effective thermal conductivity of packed pebble beds, with emphasis on the near-wall region", Nuclear Engineering and Design, vol. 331, pp. 248 $262,2018$. 
[9] S. Ergun and A.A. Orning, "Fluid flow through randomly packed columns and fluidized beds", Industrial and Engineering Chemistry, vol. 41, pp. 1179 - 1184, 1949.

[10] J. Gan, Z. Zhou and A. Yu, "Particle scale study of heat transfer in packed and fluidized beds of ellipsoidal particles", Chemical Engineering Science, vol. 144, pp. 201 - 215, 2016.

[11] G.H. Bagheri, C. Bonadonna, I. Manzella and P. Vonlanthen, "On the characterization of size and shape of irregular particles", Powder Technology, vol. 270, pp. 141-153, 2015.

[12] M.A. Taylor, E.J. Garboczi, S.T. Erdogan and D.W. Fowler, "Some properties of irregular 3D particles", Powder Technology, vol. 162, pp. $1-15,2006$.

[13] A.H. Thaker, G.M. Karthik and V.V. Buwa, "PIV measurements and CFD simulations of the particle-scale flow distribution in a packed bed", Chemical Engineering Journal, vol. 374, pp. 189 - 200, 2019.

[14] W.D. Fullmer and J. Musser, "CFD-DEM solution verification: fixed bed studies", Powder Technology, vol. 339, pp. $760-764,2018$.

[15] H.P. Zhu, Z.Y. Zhou, R.Y. Yang and A.B. Yu, "Discrete particle simulation of particulate systems: A review of major applications and findings", Chemical Engineering Science, vol. 63, pp. 5728 - 5770, 2008.

[16] B. Partopour and A.G. Dixon, "An integrated workflow for resolved-particle packed bed models with complex particle shapes", Powder Technology, vol. 322, pp. 258 - 272, 2017.

[17] J. Linsong, L. Hongsheng, W. Dan, W. Jiansheng and X. Maozhao, "Pore-scale simulation of vortex characteristics in randomly packed beds using LES/RANS models", Chemical Engineering Science, vol. 177, pp. 431 - 444, 2018.

[18] V.A. Patil and J.A. Liburdy, "Scale estimation for turbulent flows in porous media", Chemical Engineering Science, vol. 123, pp. $231-235,2015$.

[19] L.W. Rong, Z.Y. Zhou and A.B. Yu, "Lattice-Boltzmann simulation of flow through packed beds of uniform ellipsoids", Powder Technology, vol. 285, pp. 146 - 156, 2015.

[20] X. Wang, F. Thauvin and K.K. Mohanty, "Non-Darcy flow through anisotropic media", Chemical Engineering Science, vol. 54, pp. 1859 - 1869, 1999.

[21] X. Li, M. Jiang, Z. Huang and Q. Zhou, "Effect of particle orientation on the drag force of random arrays of prolate ellipsoids in low Reynolds number flows", AIChE Journal, vol. 65, 2019.

[22] M.R. Spiegel, "Mathematical handbook of formulas and tables", McGraw-Hill, 1968.

[23] F.N. Okonta and S.G. Magalula, "Railway foundation properties of some South African quarry stones", The Electronic Journal of Geotechnical Engineering, vol. 16B, pp. 179 - 197, 2011.

[24] D.W. Waples and J.S. Waples, "A review and evaluation of specific heat capacities of rocks, minerals, and subsurface fluids. Part 1: minerals and nonporous rocks", Natural Resources Research, vol. 13, pp. 97 - 122, 2004.

[25] S. Lommen, D. Schott and G. Lodewijks, "DEM speedup: Stiffness effects on behaviour of bulk material", Particuology, vol. 12, pp. 107 - 112, 2014.

[26] K. Taira and T. Colonius, "The immersed boundary method: a projection approach", Journal of Computational Physics, vol. 225, pp. $2118-2137,2007$.

[27] J.E. Hoffmann and P.J. Lindeque, "Pressure drop through randomly packed beds of ellipsoidal particles", in Proceedings of HEFAT 2019, Wicklow, Ireland, 2019. 